\title{
Prediction of treatment-induced changes in target-organ damage using changes in clinic, home and ambulatory blood pressure
}

\author{
Nikos Karpettas ${ }^{1}$, Antonis Destounis ${ }^{1}$, Anastasios Kollias ${ }^{1}$, Efthimia Nasothimiou ${ }^{1}$, Ioannis Moyssakis ${ }^{2}$ \\ and George S Stergiou ${ }^{1}$
}

Cross-sectional studies have shown that ambulatory and home blood pressure (ABP and HBP, respectively) measurements are more closely associated with preclinical organ damage than are office measurements. This study investigated the association between treatment-induced changes in BP assessed by the three methods and the corresponding changes in organ damage. Untreated hypertensives were evaluated with office, ABP and HBP measurements and indices of organ damage (echocardiographic left-ventricular mass index (LVMI), pulse wave velocity (PWV), albuminuria) before and after 12 months of treatment. A total of 116 subjects completed the study (mean age $50.7 \pm 10.5$ years, 69 men (59\%), mean follow-up $13.4 \pm 1.4$ months). The treatment-induced change in the LVMI was correlated with changes in BP and pulse pressure (PP) assessed by all methods. The change in PWV was correlated with changes in home systolic and ABP and PP and with the change in home diastolic BP. Albuminuria showed no correlations. In linear regression models, changes in home BP and PP had the strongest predictive ability for the change in the LVMI, whereas the change in ABP was the strongest predictor of the change in PWV. The change in office BP had no predictive value. HBP and ABP measurements appear to be superior to office BP measurements and should be considered complementary rather than interchangeable methods for monitoring the effects of antihypertensive treatment on target-organ damage.

Hypertension Research (2014) 37, 543-547; doi:10.1038/hr.2014.24; published online 27 February 2014

Keywords: ambulatory blood pressure; home blood pressure; left ventricular hypertrophy; self-measurement; target-organ damage

\section{INTRODUCTION}

Blood pressure (BP) measurement by a doctor in the office or clinic has been regarded for decades as the cornerstone of hypertension diagnosis and monitoring treatment-induced BP reductions. However, these measurements often do not accurately reflect the 'true' BP level and target-organ status ${ }^{1}$ because of the white coat and masked hypertension phenomena, the poor reproducibility of office measurements and observer prejudice and bias. ${ }^{1}$ Several studies have shown that out-of-office measurements of BP either at home (HBP) or with 24-h ambulatory monitoring (ABP) are more reproducible than office measurements and more accurately reflect preclinical target-organ damage ${ }^{2}$ and the risk of cardiovascular events. ${ }^{3,4}$

Both ABP and HBP monitoring are widely used in clinical practice and are endorsed by the current hypertension guidelines. ${ }^{5,6}$ Several studies have shown that the two methods have similar abilities to detect the white coat and masked hypertension phenomena in both untreated and treated subjects. ${ }^{7,8}$ Moreover, several comparative cross-sectional studies have shown similar associations of ABP and HBP measurements with indices of preclinical organ damage. ${ }^{2}$
However, limited evidence exists on the association between treatment-induced changes in ABP versus $\mathrm{HBP}$ and treatmentinduced changes in target-organ damage. ${ }^{9}$

This study investigated the associations between the treatmentinduced changes in BP assessed by office, home and ambulatory measurements and the corresponding treatment-induced changes in indices of preclinical target-organ damage.

\section{METHODS}

Participants

Adults aged $>30$ years who were referred to a hospital outpatient hypertension clinic for elevated BP and were either previously untreated or treated for $<2$ weeks were invited to participate. The exclusion criteria were clinic systolic $\mathrm{BP} \geqslant 180 \mathrm{~mm} \mathrm{Hg}$ and/or diastolic $\geqslant 110 \mathrm{~mm} \mathrm{Hg}$, evidence of secondary hypertension, sustained arrhythmia, pregnancy, history of coronary heart disease, heart failure or stroke, serum creatinine $>2 \mathrm{mg} \mathrm{dl}^{-1}$, proteinuria, uncontrolled diabetes (hemoglobin $A_{1} c>8 \%$ ), use of any drugs known to affect BP (excluding aspirin up to $300 \mathrm{mg} \mathrm{day}^{-1}$ ), any acute severe chronic disease and inability to self-monitor BP at home. The study protocol was 
approved by the hospital scientific committee, and all participants signed informed consent (trial registration at ClinicalTrials.gov NCT00841308).

\section{Design}

Subjects who fulfilled the study entry criteria were enrolled and participated in a 2-week run-in period during which they were evaluated with the following: (i) measurements of BP in the clinic, at home and with ambulatory monitoring; (ii) laboratory investigations (full blood count and routine blood biochemistry, including glucose, urea, electrolytes, liver function tests, lipid profile, urine microscopy and dipstick); and (iii) assessment of preclinical target-organ damage (electrocardiogram, echocardiography, carotid-femoral pulse wave velocity (PWV) and urinary albumin excretion (UAE)). Participants who were previously treated entered the run-in period if their clinic BP was above target (see below) after a 4 -week washout period or if their clinic BP was $\geqslant 160 / 100 \mathrm{~mm} \mathrm{Hg}$ after the 2-week washout.

According to the European Society of Hypertension recommendations at the time the study began, ${ }^{10}$ the participants were classified as having low-moderate or high cardiovascular risk (diabetes mellitus, established cardiovascular disease, preclinical target-organ damage or the presence of $\geqslant 3$ cardiovascular risk factors). ${ }^{10}$ Consequently, the $\mathrm{BP}$ targets for low-moderate risk patients were defined as $<140 / 90 \mathrm{~mm} \mathrm{Hg}$ for clinic BP and $<135 / 85 \mathrm{~mm} \mathrm{Hg}$ for HBP and awake ABP, whereas for high-risk patients the BP targets were $<130 / 80$ and $<125 / 80 \mathrm{~mm} \mathrm{Hg}$, respectively.

In subjects who fulfilled the study selection criteria and had a BP above target by one or more of the measurement methods (clinic, home, ambulatory), antihypertensive treatment was administered using a stepwise add-on treatment strategy (see below) until the above-mentioned BP goals were reached. Treatment initiation and titration were based on either clinic BP and ABP measurement or HBP measurement alone, with participants randomized to the two treatment strategy arms (the comparison between the two groups has been reported elsewhere $\left.{ }^{11}\right)$.

Controlled patients were followed at 6-month intervals if they were at lowmoderate cardiovascular risk and at 3-month intervals if they were at high risk. After 12 months of treatment, the participants underwent the same evaluation as at baseline, including clinic BP, HBP and ABP measurements, laboratory investigations and target-organ damage evaluation.

\section{BP measurements}

Duplicate clinic BP measurements were taken in a single study visit after $5 \mathrm{~min}$ of sitting at rest with $1 \mathrm{~min}$ between measurements using a validated professional oscillometric device (Microlife WatchBP Office, Microlife AG, Widnau, Switzerland). ${ }^{12}$ HBP monitoring was performed for 7 routine workdays within 2 weeks, with duplicate self-measurements in the morning (0600-0900 hours, before drug intake when treated) and in the evening (18002100 hours) after $5 \mathrm{~min}$ of sitting at rest with $1 \mathrm{~min}$ between measurements using a validated oscillometric device with automated memory and PC link capacity (Microlife WatchBP Home, Microlife AG) ${ }^{13}$ HBP monitoring was repeated if $<8$ valid $\mathrm{HBP}$ readings were obtained. All HBP readings were averaged after the first-day measurements were discarded. Patients were asked to fill in a form with their self-HBP measurements, and these were verified after being downloaded from the device's memory. ABP was monitored on a routine workday at 20-min intervals for $24 \mathrm{~h}$ using validated oscillometric devices: SpaceLabs 90207 or 90217 (Healthcare, Issaquah, WA, USA) ${ }^{14}$ or Microlife WatchBP O3 (Microlife AG). ${ }^{15}$ Each individual used the same type of ABP monitor throughout the study. ABP recordings with $<20$ valid awake and 7 asleep readings were repeated. Awake and asleep ABP was calculated using each individual's report for in-bed and out-of-bed periods. HBP and ABP monitoring were performed in random order according to device availability and patient's preference.

\section{Target-organ damage}

As mentioned above, assessment of preclinical target-organ damage was performed during the run-in period and at the end of follow-up. An M-mode echocardiogram was performed by a single observer (IM) who was unaware of the participant's medical history, BP measurements and treatment status. The observer was blinded to the baseline assessment when performing the final echocardiogram. Left-ventricular mass was calculated from the average of three cardiac cycles using the Devereux formula ${ }^{16}$ and was divided by body-surface area $^{17}$ to provide the left-ventricular mass index (LVMI). Arterial distensibility was assessed by measuring the carotid-femoral PWV using a Complior device (Colson, Garges-les-Gonesse, Paris, France). The device calculates PWV using a transducer positioned at the base of the neck for the common carotid artery and another over the femoral artery to record pulse waves. ${ }^{18}$ The PWV for each individual was regarded as the average of two measurements taken on a single occasion. UAE was assessed by two morning urine samples (within 7 days), and the two values were averaged for each individual for each assessment

\section{Treatment strategy}

A stepwise add-on treatment protocol was used, intensifying treatment at 4-6-week intervals until the previously specified BP goals were reached: step 1: full-dose angiotensin receptor blocker $(300 \mathrm{mg}$ o.d. irbesartan, $80 \mathrm{mg}$ o.d. telmisartan, $320 \mathrm{mg}$ o.d. valsartan); step 2: add-on $5 \mathrm{mg}$ o.d amlodipine; step 3: titration to $10 \mathrm{mg}$ o.d. amlodipine; step 4 : add-on $12.5 \mathrm{mg}$ o.d. hydrochlorothiazide; step 5: titration of hydrochlorothiazide to $25 \mathrm{mg}$ o.d. (in case of side effects e.g. edema, hypokalemia, any of steps 2 to 5 was omitted accordingly); step 6: add-on $12.5 \mathrm{mg}$ b.i.d. carvedilol; step 7: carvedilol titrated to $25 \mathrm{mg}$ b.i.d.; and step 8: add-on spironolactone or other drugs according to the doctor's discretion. Treatment of other cardiovascular risk factors with hypolipidemic, antidiabetic or antiplatelet drugs was administered according to the established guidelines.

\section{Analysis}

Participants with complete BP and target-organ damage evaluations both at baseline and at the end of follow-up were analyzed. The Kolmogorov-Smirnov test was used to check the normality of the distribution of the study samples as well as the standardized and unstandardized residuals of linear regression models. Student's paired $t$-test was used to compare BP at baseline and at the end of the study, and the Wilcoxon's test was used for paired comparisons of non-parametric data. Pearson correlations were used to assess correlations between treatment-induced changes in BP assessed by different measurement methods and the corresponding changes in indices of target-organ damage. Stepwise linear regression models were used to investigate independent predictors of the change in each of the studied indices of target-organ damage, including age, sex, body mass index and changes in clinic BP, HBP, ABP and pulse pressure $(\mathrm{PP})$, correcting for clinic $\mathrm{BP}$ at baseline. Statistical analysis was performed using the SPSS 17.0 statistical software (SPSS, Chicago, IL, USA) A $P$-value $<0.05$ was considered statistically significant

\section{RESULTS}

A total of 172 consecutive subjects were recruited; 22 were treated for up to 2 weeks and invited to enter the study after a 2-4-week washout period. Twenty-seven subjects were excluded (13 were normotensive; 8 failed or refused to complete the run-in assessment; 4 treated subjects failed to complete the washout period; 1 had obstructive sleep apnea; and 1 had stage 3 hypertension). Therefore, 145 subjects were enrolled in the study, and antihypertensive drug treatment was initiated. Twenty-nine subjects $(20 \%)$ were withdrawn due to incomplete follow-up, and 116 subjects completed the study and were included in the analysis. There was no difference between subjects who dropped out versus those analyzed regarding age, gender, body mass index, clinic BP, HBP, systolic ABP, LVMI or PWV. However, dropouts had lower awake diastolic ABP $(P<0.05)$ and higher UAE $(P<0.05)$

The mean age of the 116 subjects included in the analysis was $50.7 \pm 10.5$ years, the mean body mass index was $28.9 \pm 4.2 \mathrm{~kg} \mathrm{~m}^{-2}$ and the mean waist circumference was $101.6 \pm 13.2 \mathrm{~cm}$. Sixty-nine of the subjects were men (59\%), and 34 were smokers (29\%). Three patients had diabetes mellitus (2.6\%), 100 had dyslipidemia (86\%) and 55 were at high cardiovascular risk (47\%). The mean follow-up period was $13.4 \pm 1.4$ months. The BP levels assessed by each 
Table 1 Blood pressure levels and target-organ damage at baseline and at the end of follow-up (13.4 \pm 1.4 months)

\begin{tabular}{|c|c|c|}
\hline & Baseline & Decline \\
\hline \multicolumn{3}{|c|}{ Blood pressure $(\mathrm{mm} \mathrm{Hg})$ systolic/diastolic } \\
\hline Clinic & $146.6 \pm 12.6 / 96.3 \pm 8.1$ & $17.0 \pm 14.4^{*} / 12.3 \pm 8.6^{*}$ \\
\hline Home & $141.2 \pm 11.8 / 89.6 \pm 7.7$ & $15.9 \pm 12.6 * / 9.4 \pm 7.4^{*}$ \\
\hline 24-h & $134.3 \pm 11.2 / 84.4 \pm 7.7$ & $15.7 \pm 12.0 * / 10.3 \pm 7.6^{*}$ \\
\hline Awake & $140.3 \pm 11.8 / 89.5 \pm 8.3$ & $15.9 \pm 13.3 * / 10.6 \pm 8.7 *$ \\
\hline Asleep & $122.9 \pm 12.8 / 75.0 \pm 8.7$ & $14.2 \pm 12.2 * / 8.8 \pm 8.4^{*}$ \\
\hline \multicolumn{3}{|c|}{ Pulse pressure $(\mathrm{mm} \mathrm{Hg})$} \\
\hline Clinic & $50.3 \pm 10.4$ & $4.7 \pm 10.7 *$ \\
\hline Home & $51.6 \pm 9.2$ & $6.5 \pm 6.8^{*}$ \\
\hline 24-h & $49.9 \pm 8.2$ & $5.4 \pm 6.7^{*}$ \\
\hline Awake & $50.8 \pm 8.6$ & $5.3 \pm 7.3^{*}$ \\
\hline Asleep & $47.9 \pm 8.8$ & $5.4 \pm 7.0^{*}$ \\
\hline \multicolumn{3}{|l|}{ Target-organ damage } \\
\hline IVS (mm) & $10.60 \pm 0.70$ & $0.17 \pm 0.34^{*}$ \\
\hline LVM (g) & $199.8 \pm 26.0$ & $3.7 \pm 10.3^{*}$ \\
\hline LVM index $\left(\mathrm{g} \mathrm{m}^{-2}\right)$ & $100.8 \pm 10.3$ & $2.7 \pm 5.6^{*}$ \\
\hline $\mathrm{PWV}\left(\mathrm{ms}^{-1}\right)$ & $7.29 \pm 1.84$ & $0.73 \pm 1.89 *$ \\
\hline UAE (mg dl $\left.{ }^{-1}\right)$ & $10.5 \pm 19.8$ & $0.9 \pm 11.6$ \\
\hline
\end{tabular}

Abbreviations: BP, blood pressure; IVS, intraventricular septum; LVM, left ventricular mass; PWV, pulse wave velocity; UAE, urinary albumin excretion.

${ }^{*} P<0.001$.

measurement method and the indices of target-organ damage at baseline are presented in Table 1. Eleven subjects (9.5\%) had left ventricular hypertrophy at baseline according to the accepted cutoff values at the time the study was designed $\left(125 \mathrm{~g} \mathrm{~m}^{-2}\right.$ for men and $110 \mathrm{~g} \mathrm{~m}^{-2}$ for women), ${ }^{10}$ whereas according to the 2013 guidelines of the European Society of Hypertension ${ }^{19}$ (cutoff values of $115 \mathrm{~g} \mathrm{~m}^{-2}$ and $95 \mathrm{~g} \mathrm{~m}^{-2}$, respectively), $34(29.3 \%)$ participants had left ventricular hypertrophy. Furthermore, $8(7 \%)$ had elevated PWV $\left(>10 \mathrm{~m} \mathrm{~s}^{-1}\right) \cdot{ }^{20}$ At the end of follow-up, there were significant reductions in BP, as assessed by all three measurement methods, as well as in LVMI and PWV; no reduction was observed in UAE (Table 1). The decline in clinic BP was only slightly greater than that in $\mathrm{ABP}$ and HBP, which is most likely because the study participants were referred to a hypertension center and had previous office visits, which reduced their office $\mathrm{BP}$, and because the office measurements were performed in a research setting and were therefore strictly standardized.

The associations of treatment-induced changes in BP and PP assessed by the three measurement methods with the corresponding treatment-induced changes in indices of target-organ damage are presented in Table 2. The treatment-induced change in LVMI was significantly correlated with the changes in BP (systolic and diastolic) and $\mathrm{PP}$ assessed by clinic, home and ambulatory monitoring, with the exception of nighttime diastolic ABP. Similar correlations were found for changes in left ventricular mass and intraventricular septum thickness with changes in BP and PP (data not shown). The treatment-induced change in PWV was significantly correlated with the changes in (a) systolic BP assessed by all methods, excluding clinic BP, (b) diastolic BP assessed only at home and (c) PP assessed by all methods, excluding clinic BP. The treatment-induced change in UAE showed no significant correlations with systolic or diastolic BP or PP assessed by any of the measurement methods (data not shown). There was a trend toward HBP being more closely associated with the LVMI
Table 2 Pearson correlation coefficients $(r)$ between treatmentinduced changes in clinic, home and ambulatory blood pressure and pulse pressure with the changes in left ventricular mass index and pulse wave velocity

\begin{tabular}{|c|c|c|c|c|c|c|}
\hline \multirow{2}{*}{$\begin{array}{l}\text { Measurement } \\
\text { method }\end{array}$} & \multicolumn{2}{|c|}{$\triangle$ Systolic BP } & \multicolumn{2}{|c|}{$\triangle$ Diastolic BP } & \multicolumn{2}{|c|}{$\triangle$ Pulse pressure } \\
\hline & $\triangle L V M I$ & $\triangle P W V$ & $\triangle L V M I$ & $\triangle P W V$ & $\triangle L V M I$ & $\triangle P W V$ \\
\hline Clinic & $0.38^{* *}$ & 0.13 & $0.33^{* *}$ & 0.14 & $0.24^{*}$ & 0.07 \\
\hline Home & $0.44^{* *}$ & $0.36^{* *}$ & $0.42^{* *}$ & $0.33^{* *}$ & $0.36^{* *}$ & $0.32^{* *}$ \\
\hline 24-h & $0.37^{* *}$ & $0.28 *$ & $0.26^{*}$ & 0.10 & $0.34^{* *}$ & $0.38^{* *}$ \\
\hline Awake & $0.36^{* *}$ & $0.26^{*}$ & $0.29^{* *}$ & 0.13 & $0.29^{* *}$ & $0.30^{* *}$ \\
\hline Asleep & $0.29^{* *}$ & $0.36^{* *}$ & 0.12 & 0.13 & $0.32^{* *}$ & $0.36^{* *}$ \\
\hline
\end{tabular}

Abbreviations: $\Delta$, treatment-induced change; BP, blood pressure; LVMI, left ventricular mass index; PWV, pulse wave velocity.

${ }^{*} P<0.05 ;{ }^{* *} P<0.01$

and PWV than the clinic BP or ABP measurements, but the differences in correlation coefficients did not reach a statistical significance (Table 2).

Stepwise linear regression models (Table 3) were tested using age, sex, body mass index and baseline systolic clinic BP as independent variables and the change in systolic and diastolic BP according to all three methods (model A), the change in PP (model B) or both (model C) as dependent variables. In model A, the change in systolic HBP predicted the LVMI and PWV changes, and changes in 24-h and asleep ABP predicted the change in PWV. In model B, changes in home and asleep PP predicted the LVMI change, and the change in 24-h PP predicted the PWV change. In model C, changes in systolic HBP and asleep PP predicted the LVMI change, and the change in 24-h PP predicted the PWV change. The change in clinic BP or PP was not an independent predictor of the change in either the LVMI or the PWV. The change in UAE also did not appear to have independent predictive value. Tolerance ranged between 0.47 and 1.0 and variance inflation factor (VIF) between 1.0 and 2.1. The standardized and unstandardized residuals of all models showed normal distributions according to the Kolmogorov-Smirnov test.

\section{DISCUSSION}

One of the recognized advantages of out-of-office BP measurements compared with conventional office measurements is their closer association with indices of preclinical organ damage. The vast majority of the studies that have demonstrated this relationship had cross-sectional designs for both $\mathrm{ABP}^{5}$ and $\mathrm{HBP}$ measurements. ${ }^{2,6}$ However, the crucial question is whether treatment-induced changes in $\mathrm{HBP}$ or ABP more accurately reflect the treatment-induced changes in organ damage than do office BP measurements.

To our knowledge, only a single study has previously investigated this research question using office, $\mathrm{HBP}$ and $\mathrm{ABP}$ measurements (Study on Ambulatory Monitoring of Blood Pressure and Lisinopril Evaluation (SAMPLE)). ${ }^{9}$ The study included 206 hypertensives followed for 12 months, and the regression of left ventricular hypertrophy was more closely associated with treatment-induced changes in ABP than with changes in office BP or even HBP. However, in the SAMPLE study, the potential of HBP was not exhausted because only two home readings were obtained. Current guidelines recommend evaluating $\mathrm{HBP}$ by averaging readings taken over 7 days (at least three) after discarding those taken on the first day, which are known to be higher and more unstable (higher SD); ${ }^{6}$ in fact those readings investigated in the SAMPLE study. 
Table 3 Multivariable analysis of variables independently associated with treatment-induced changes in left ventricular mass index and pulse wave velocity (linear regression models using stepwise method)

\begin{tabular}{|c|c|c|c|c|c|c|}
\hline \multirow[b]{2}{*}{ Independent variables } & \multicolumn{3}{|c|}{$\Delta$ Left ventricular mass index } & \multicolumn{3}{|c|}{$\triangle$ Pulse wave velocity } \\
\hline & Associated variables & $\mathrm{R}^{2}$ & $\beta$ & Associated variables & $\mathrm{R}^{2}$ & $\beta$ \\
\hline \multirow[t]{3}{*}{ Model A: Age, sex, BMI, baseline clinic SBP, and $\Delta$-BP } & $\Delta$ Home SBP & 0.192 & 0.439 & $\Delta$ Home SBP & 0.130 & 0.335 \\
\hline & & & & $\Delta$ Asleep SBP & 0.183 & 0.511 \\
\hline & & & & $\Delta$ 24-h DBP & 0.263 & -0.412 \\
\hline \multirow[t]{2}{*}{ Model B: Age, sex, BMI, baseline clinic SBP, and $\Delta$-PP } & $\Delta$ Home PP & 0.129 & 0.303 & $\Delta$ 24-h PP & 0.144 & 0.379 \\
\hline & $\Delta$ Asleep PP & 0.191 & 0.256 & & & \\
\hline \multirow[t]{2}{*}{ Model C: Age, sex, BMI, baseline clinic SBP, $\Delta$-BP and $\Delta$-PP } & $\Delta$ Home SBP & 0.192 & 0.379 & $\Delta$ 24-h PP & 0.144 & 0.379 \\
\hline & $\Delta$ Asleep PP & 0.236 & 0.218 & & & \\
\hline
\end{tabular}

Abbreviations: $\Delta$, treatment-induced change; $\Delta$-BP, treatment-induced change in systolic and diastolic blood pressure by all measurement methods; $\Delta$-PP, treatment-induced change in pulse pressure by all measurement methods; BMI, body mass index; DBP, diastolic blood pressure; PP, pulse pressure; SBP, systolic blood pressure.

The present study examined the association between treatmentinduced changes in BP and treatment-induced changes in targetorgan damage after an average follow-up of 13.4 months. The main advantages of the study are that a direct comparison of the three BP measurement methods (clinic, home, ambulatory) was performed in the same subjects, the measurement methods were applied according to current guidelines ${ }^{5,6}$ and three indices of preclinical organ damage were evaluated.

The main findings of the study are that (i) both HBP and ABP are superior to the conventional clinic measurements in predicting treatment-induced target-organ damage, (ii) HBP appears to be at least as good as ABP in this respect, and (iii) treatment-induced changes in different indices of target-organ damage might differ in their association with the changes in BP evaluated by different measurement methods (HBP or ABP).

As demonstrated by the linear regression models (Table 3), the treatment-induced changes in home BP and PP appear to be the most reliable predictors of treatment-induced changes in LVMI. However, treatment-induced changes in ambulatory BP and PP appeared to be the most efficient method for predicting treatment-induced changes in PWV. These findings suggest that HBP and ABP might not be fully interchangeable methods in the evaluation of out-of-office BP in treated hypertensives and the effects of treatment on the heart and vasculature. $\mathrm{HBP}$ and $\mathrm{ABP}$ have important similarities, because they both provide multiple BP measurements away from the clinic in the usual environment of each individual. Nonetheless, they also have major differences; HBP is monitored for several days, weeks or even months, but only at home and in a sitting position, whereas $\mathrm{ABP}$ is monitored for only $24 \mathrm{~h}$ in different conditions in terms of environment, posture and activity, including at work, at home and even during sleep.

These data support the view that $\mathrm{HBP}$ and $\mathrm{ABP}$ are complementary rather than competitive methods for the evaluation of out-of-office BP. This view is supported by a recent analysis of the PAMELA general population study, which demonstrated that among subjects with white coat hypertension (elevated office BP and low HBP or $\mathrm{ABP}$ ), those with both low HBP and low ABP had lower cardiovascular mortality than subjects in whom only one or the other measurement was low. ${ }^{21}$ The authors of this paper concluded that both HBP and ABP monitoring are required to identify accurately the white coat hypertension phenomenon. ${ }^{21}$

This is an analysis of data obtained from a study that aimed to compare two hypertension treatment strategies, and the participants were recruited on the basis of elevated BP rather than the presence of organ damage. This recruitment strategy explains the moderate organ damage exhibited by the participants and the low prevalence of left ventricular hypertrophy, which are limitations of the study.

In conclusion, $\mathrm{HBP}$ and $\mathrm{ABP}$ are superior to conventional office $\mathrm{BP}$ in monitoring the effects of antihypertensive drug treatment on target-organ damage. These methods appear to be complementary rather than competitive, and their combined application appears to be the optimal approach for the precise evaluation of the impact of treatment on hypertension-induced target-organ damage.

\section{CONFLICT OF INTEREST}

GSS has received consulting fees from Microlife, Widnau, Switzerland. The other authors declare no conflict of interest.

\section{ACKNOWLEDGEMENTS}

Some of the blood pressure monitors used in this study were provided by Microlife, Widnau, Switzerland.

1 O'Brien E, Asmar R, Beilin L, Imai Y, Mallion JM, Mancia G, Mengden T, Myers M, Padfield P, Palatini P, Parati G, Pickering T, Redon J, Staessen J, Stergiou G, Verdecchia P. European Society of Hypertension recommendations for conventional, ambulatory and home blood pressure measurement. J Hypertens 2003; 21: 821-848.

2 Bliziotis IA, Destounis A, Stergiou GS. Home versus ambulatory and office blood pressure in predicting target organ damage in hypertension: a systematic review and meta-analysis. J Hypertens 2012; 30: 1289-1299.

3 Stergiou GS, Siontis KC, loannidis JP. Home blood pressure as a cardiovascular outcome predictor: it's time to take this method seriously. Hypertension 2010; 55: 1301-1303.

4 Ward AM, Takahashi O, Stevens R, Heneghan C. Home measurement of blood pressure and cardiovascular disease: systematic review and meta-analysis of prospective studies. J Hypertens 2012; 30: 449-456.

5 O'Brien E, Parati G, Stergiou G, Asmar R, Beilin L, Bilo G, Clement D, de la Sierra A, de Leeuw P, Dolan E, Fagard R, Graves J, Head G, Imai Y, Kario K, Lurbe E, Mallion JM, Mancia G, Mengden T, Myers M, Ogedegbe G, Ohkubo T, Omboni S, Palatini P, Redon J, Ruilope LM, Shennan A, Staessen JA, vanMontfrans G, Verdecchia P, Waeber B, Wang J, Zanchetti A, Zhang Y, on behalf of the European Society of Hypertension Working Group on Blood Pressure Monitoring. European Society of Hypertension position paper on ambulatory blood pressure monitoring. J Hypertens 2013; 31: 1731-1768.

6 Parati G, Stergiou GS, Asmar R, Bilo G, de Leeuw P, Imai Y, Kario K, Lurbe E, Manolis A, Mengden T, O'Brien E, Ohkubo T, Padfield P, Palatini P, Pickering T, Redon J, Revera M, Ruilope LM, Shennan A, Staessen JA, Tisler A, Waeber B, Zanchetti A, Mancia G. European Society of Hypertension guidelines for blood pressure monitoring at home: a summary report of the second international consensus conference on home blood pressure monitoring. J Hypertens 2008; 26: 1505-1526.

7 Stergiou GS, Bliziotis IA. Home blood pressure monitoring in the diagnosis and treatment of hypertension: a systematic review. Am J Hypertens 2011; 24: 123-134.

8 Nasothimiou EG, Tzamouranis D, Rarra V, Roussias LG. Stergiou GS. Diagnostic accuracy of home vs. ambulatory blood pressure monitoring in untreated and treated hypertension. Hypertens Res 2012; 35: 750-755. 
9 Mancia G, Zanchetti A, Agabiti-Rosei E, Benemio G, De Cesaris R, Fogari R, Pessina A, Porcellati C, Rappelli A, Salvetti A, Trimarco B. Ambulatory blood pressure is superior to clinic blood pressure in predicting treatment-induced regression of left ventricular hypertrophy. SAMPLE Study Group. Study on Ambulatory Monitoring of Blood Pressure and Lisinopril Evaluation. Circulation 1997; 95: 1464-1470.

10 Mancia G, De Backer G, Dominiczak A, Cifkova R, Fagard R, Germano G, Grassi G, Heagerty AM, Kjeldsen SE, Laurent S, Narkiewicz K, Ruilope L, Rynkiewicz A, Schmieder RE, Boudier HA, Zanchetti A, Vahanian A, Camm J, De Caterina R, Dean V, Dickstein K, Filippatos G, Funck-Brentano C, Hellemans I, Kristensen SD, McGregor K, Sechtem U, Silber S, Tendera M, Widimsky P, Zamorano JL, Erdine S, Kiowski W, Agabiti-Rosei E, Ambrosioni E, Lindholm LH, Viigimaa M, Adamopoulos S, Agabiti-Rosei E, Ambrosioni E, Bertomeu V, Clement D, Erdine S, Farsang C, Gaita D, Lip G, Mallion JM, Manolis AJ, Nilsson PM, O'Brien E, Ponikowski P, Redon J, Ruschitzka F, Tamargo J, van Zwieten P, Waeber B, Williams B, Management of Arterial Hypertension of the European Society of Hypertension; European Society of Cardiology. 2007 Guidelines for the Management of Arterial Hypertension: The Task Force for the Management of Arterial Hypertension of the European Society of Hypertension and of the European Society of Cardiology. J Hypertens 2007; 25: 1105-1187.

11 Stergiou G, Karpettas N, Destounis A, Tzamouranis D, Nasothimiou E, Kollias A, Roussias L, Moyssakis I. Home blood pressure monitoring alone versus combined clinic and ambulatory measurements in following treatment induced changes in blood pressure and organ damage. Am J Hypertens 2014; 27: 184-192.

12 Stergiou GS, Tzamouranis D, Protogerou A, Nasothimiou E, Kapralos C. Validation of the Microlife Watch BP Office professional device for office blood pressure measurement according to the International Protocol. Blood Press Monit 2008; 13: 299-303.

13 Stergiou GS, Giovas PP, Gkinos CP, Patouras JD. Validatin of the Microlife WatchBP Home device for self home blood pressure measurement according to the International Protocol. Blood Press Monit 2007; 12: 185-188.

14 O'Brien E, Mee F, Atkins N, Halligan A, O'Malley K. Accuracy of the SpaceLabs 90207 ambulatory blood pressure measuring system in normotensive pregnant women determined by the British Hypertension Society protocol. J Hypertens 1993; 11: S282-S283

15 Ragazzo F, Saladini F, Palatini P. Validation of the Microlife WatchBP 03 device for clinic, home, and ambulatory blood pressure measurement, according to the International Protocol. Blood Press Monit 2010; 15: 59-62.
16 Devereux RB, Alonso DR, Lutas EM, Gottlieb GJ, Campo E, Sachs I, Reichek N. Echocardiographic assessment of left ventricular hypertrophy: comparison to necropsy findings. Am J Cardiol 1986; 57: 450-458.

17 Mosteller RD. Simplified calculation of body-surface area. New Eng/ J Med 1987; 317: 1098.

18 Mattace-Raso F, Hofman A, Verwoert GC, Wittemana JC, Wilkinson I, Cockcroft J, McEniery C, Yasmin, Laurent S, Boutouyrie P, Bozec E, Hansen TW, Torp-Pedersen C, Ibsen H, Jeppesen J, Vermeersch SJ, Rietzschel E, De Buyzere M, Gillebert TC, Van Bortel L, Segers P, Vlachopoulos C, Aznaouridis C, Stefanadis C, Benetos A, Labat C, Lacolley P, Stehouwer C, Nijpels G, Dekker JM, Stehouwer C, Ferreira I, Twisk JW, Czernichow S, Galan P, Hercberg S, Pannier B, Guérin A, London G, Cruickshank JK, Anderson SG, Paini A, Agabiti Rosei E, Muiesan ML, Salvetti M, Filipovsky J, Seidlerova J, Dolejsova M. Determinants of pulse wave velocity in healthy people and in the presence of cardiovascular risk factors: establishing normal and reference values. Reference values for Arterial Stiffness' Collaboration. Eur Heart $J$ 2010; 31: 2338-2350.

19 Mancia G, Fagard R, Narkiewicz K, Redón J, Zanchetti A, Böhm M, Christiaens T, Cifkova R, De Backer G, Dominiczak A, Galderisi M, Grobbee DE, Jaarsma T, Kirchhof P, Kjeldsen SE, Laurent S, Manolis AJ, Nilsson PM, Ruilope LM, Schmieder RE, Sirnes PA, Sleight P, Viigimaa M, Waeber B, Zannad F, Task Force Members. 2013 ESH/ESC Guidelines for the management of arterial hypertension: the Task Force for the management of arterial hypertension of the European Society of Hypertension (ESH) and of the European Society of Cardiology (ESC). J Hypertens 2013; 31: 1281-1357.

20 Van Bortel LM, Laurent S, Boutouyrie P, Chowienczyk P, Cruickshank JK, De Backer T, Filipovsky J, Huybrechts S, Mattace-Raso FU, Protogerou AD, Schillaci G, Segers P, Vermeersch S, Weber T, Artery Society; European Society of Hypertension Working Group on Vascular Structure and Function; European Network for Noninvasive Investigation of Large Arteries. Expert consensus document on the measurement of aortic stiffness in daily practice using carotid-femoral pulse wave velocity. $J$ Hypertens 2012; 30: 445-448.

21 Mancia G, Bombelli M, Brambilla G, Facchetti R, Sega R, Toso E, Grassi G. Long-term prognostic value of white coat hypertension: an insight from diagnostic use of both ambulatory and home blood pressure measurements. Hypertension 2013; 62 : 168-174. 